https://doi.org/10.48009/1_iis_2005_28-34

\title{
REDUCING SPREADSHEET ERROR RATES
}

\author{
Dr. Harry Benham, Montana State University, hbenham@montana.edu \\ Dr. Marc Giullian, Montana State University, mguilliam@montana.edu
}

\begin{abstract}
This study looks at the impact of spreadsheet training that includes emphasis on spreadsheet errors and their consequences in reducing spreadsheet error rates. The same spreadsheet modeling task was given to students exposed to this "error awareness" training as well as similar students exposed to structural design training. Empirical results indicate that students exposed to the "error awareness" approach produced fewer errors than students exposed to the other training method. Additionally, the study indicates that more knowledge of the problem domain is associated with fewer spreadsheet errors.
\end{abstract}

Keywords: Spreadsheet errors, error rates, domain knowledge, error awareness, structural design.

\section{INTRODUCTION}

Spreadsheet software is widely credited with having been the application responsible for the penetration of personal computers into the business environment. Spreadsheet modeling is now a vital business skill and spreadsheet models are widely used as decision support tools. However these spreadsheets applications introduce the possibility of substantial errors. A Florida based construction company lost $\$ 254,000$ by relying upon an incorrect spreadsheet [8]. Spreadsheet audits and experiments involving both students and professionals have consistently found significant error rates $[4,7]$. A recent audit of the 30 most financially significant spreadsheets in an Australian consulting firm found material errors in $100 \%$ of the spreadsheets audited [3]. Given the pervasive use of spreadsheets, it is critical that spreadsheet errors be controlled.

Spreadsheet errors have been subjected to academic investigation by many authors. Panko [6] maintains a comprehensive and current review and summary online of what is know about spreadsheet error. Approaches to controlling spreadsheet error rates have tended to borrow from the software engineering literature stressing structural design elements of the spreadsheet $[1,2]$ or code inspection [7]. Unfortunately, the evidence to date suggests that these approaches are not very effective $[2,7]$.

The purpose of this paper is to introduce a new technique for training end-user spreadsheet developers and to examine its effectiveness. This paper compares the effectiveness in reducing spreadsheet errors of a structural design approach with an error awareness approach. In the next section, we describe these two alternative approaches to developing spreadsheets. It is followed by a description of the experiment performed and specific hypotheses to be tested. We then present empirical results suggesting that the error awareness approach is superior in reducing spreadsheet errors. A final section discusses conclusions and limitations of this study. 


\section{SPREADSHEET DEVELOPMENT APPROACHES}

For years, our teaching of spreadsheet modeling has emphasized structural design elements and inspection of cell contents as a spreadsheet verification technique. Anecdotal evidence suggests that the number of errors continues to be about the same. A simple alternative was to enhance students' awareness of spreadsheet errors and their consequences.

\section{Structural Design Approach}

In teaching spreadsheet modeling, the structural design approach consists of providing students with spreadsheet development guidelines. These guidelines call for students to create an introductory documentation section where in addition to basic names and dates, students are required to explain the purpose of their spreadsheet. The guidelines also call for an input section in which all input values are entered. Next is a model section carrying out calculations required by the model and displaying results. The final required section is a verification section, in which students demonstrate that their spreadsheets calculate the correct values.

\section{Error Awareness Approach}

The error awareness approach attempts to sensitize students to spreadsheet errors. This approach abandons the formal development guidelines, although separation of input data from calculations is still recommended. Spreadsheet error statistics and stories are shared with students. Students are also given two spreadsheets purporting to model the same process but yielding different numeric results. They are asked to determine which spreadsheet is "correct."

\section{EXPERIMENT AND HYPOTHESES}

Panko and Sprague [7] developed a simple modeling exercise for studying spreadsheet errors. We replicated their technique with only very minor modifications. The task given students was to construct a spreadsheet for the following problem:

You are to build a spreadsheet model to help you create a bid to build a wall. You will offer two options-rock or brick. Both walls will be built by crews of two. Crews will work three eight-hour days to build either type of wall. The wall we be 20 feet long, 6 feet tall, and 2 feet thick. Wages will be $\$ 10$ per hour per person. You will have to add $20 \%$ to wages to cover fringe benefits. Rock will cost $\$ 3$ per cubic foot. Brick will cost $\$ 2$ per cubic foot. Your bid must add a profit margin of $30 \%$ to your expected cost.

Still following Panko and Sprague [7], students were asked to respond to the following questions:

1. I would rate my expertise level in using Excel as: ("Low/Novice" to "High/Expert").

2. In completing the "Wall Building Bid" spreadsheet, I would rate the adequacy of my knowledge as: ("Very Inadequate" to "More than Adequate")

3. The "Wall Building Bid" spreadsheet was difficult for me: ("Disagree" to "Agree") 
4. In completing the "Wall Building Bid" spreadsheet, I would say that I : (Blew it Off to "Tried my Best")

5. Counting the time you've spent developing, auditing, or being trained in Excel, which category below best describes your experience?

The first four questions were scored on a Likert like scale from 1 to 7 . The fifth question provided a range of hours from less than 50 to in excess of 1000.

\section{Experiment}

Students in 5 sections were arbitrarily assigned either to a Structural Design treatment or an Error Awareness treatment. Given that there were 5 sections, it was not possible to perfectly balance treatment groups. After a few weeks of instruction, all students were given the above modeling problem and asked to respond to the five questions. In addition, each student's gender was recorded.

\section{Hypotheses}

The primary hypothesis of interest is whether or not the approach taken makes any difference in the frequency of student errors. We also expect, other things being equal, that the factors measured by a student's responses to the five questions above (i.e., Expertise in Excel, Knowledge, Level of Difficulty, Effort, and Experience) might also influence error rates. Phrased in terms of our expected results rather than null form, our hypotheses follow.

\section{$\mathrm{H}_{1}$ : $\quad$ Error Awareness will result in fewer spreadsheet errors.}

We argue that students who are aware that spreadsheet errors are commonplace and have seen repeated examples, will exercise greater care and have a greater tendency to check their work.

\section{$\mathrm{H}_{2}$ : Excel Expertise will be associated with fewer spreadsheet errors.} Those individuals rating themselves as having a higher level of expertise in Excel will be able to concentrate more on the problem to be modeled and less on the mechanics of using Excel as a tool, resulting in fewer errors.

\section{$\mathrm{H}_{3}$ : Knowledge of the problem domain will be associated with fewer errors.} Individuals more knowledgeable about the problem domain will better understand how to model the problem and therefore be less likely to make errors.

\section{$\mathrm{H}_{4}$ : Difficulty will be associated with more errors.} Individuals who find this problem more difficult will see the problem as more complex and that perceived complexity will be associated with more errors.

\section{H5: $_{5}$ Effort will be associated with fewer spreadsheet errors.}

Individuals who expend more effort will have devoted more attention to and tried harder to solve this problem. Paying attention and trying harder should help an individual avoid errors. 


\section{$\mathrm{H}_{6}$ : Experience will be associated with fewer spreadsheet errors.}

We argue that students who have spent more time learning about Excel will have a higher level of expertise and experience. These attributes should be associated with fewer errors

Gender (male) was included in the analysis as a control on the off chance that gender may have a systematic impact on spreadsheet errors.

\section{PROCEDURES}

As indicated above, sections were arbitrarily assigned to treatment groups. In total, 126 students developed spreadsheets to calculate a bid as indicated in the problem statement and responded to the five indicated questions.

Evaluation of the student spreadsheets followed the procedures and definitions established by Panko and Sprague [7]. Spreadsheet errors were classified as "Omit" errors - instances in which a fact explicitly given in the task statement was omitted, "Logic" errors - mistakes caused by using an inappropriate algorithm or incorrectly implementing an algorithm, and "Mechanical" errors - errors caused by simple slips such as pointing to the wrong cell, mistyping a number, typing the wrong operator, misreading a number from the task statement, and so forth. Each spreadsheet was independently evaluated by two raters. The raters then met and compared their error count and classification for each student spreadsheet. From the 126 spreadsheets evaluated, there were 4 instances of disagreement: a $96.8 \%$ agreement between the raters. The four spreadsheets upon which the raters disagreed were then jointly examined. In each case, the raters were able to agree upon error classification and count.

Error Rates for each error type were calculated. The raters supplied the count of the number of errors. The typical approach for calculating error rates has been to divide by the number of cells used. However, many cells may simply be labels. Since label cells cannot contain errors, as defined above, it does not seem appropriate to include those label cells in determining error rates. A Visual Basic for Applications program, counted the number of non-label cells used in the spreadsheet. Error rates were then calculated as the number of errors divided by the number of non-label cells.

\section{RESULTS}

Looking first at spreadsheet errors, $51 \%$ of the spreadsheets were error free. As reported in Table 1, the overall error rate was over 18\%, somewhat higher than the rate reported by Panko and Sprague [7]. However, their error rates were calculated by dividing by the total number of cells students filled in, including labels. This difference could be responsible for some of the increase in the average rates. Like Panko and Sprague [7], Omit errors were the most common, accounting for almost $60 \%$ of the errors made. Logic errors represent almost a third of the error made. Mechanical errors were relatively infrequent, comprising less than $9 \%$ of the error made. 
Table 1. Summary Statistics

\begin{tabular}{|l|c|c|}
\hline Variables & Mean & $\begin{array}{c}\text { Std } \\
\text { Dev. }\end{array}$ \\
\hline Error Rates & & \\
All & 0.184 & 0.344 \\
Omit & 0.109 & 0.238 \\
Logic & 0.058 & 0.202 \\
Mechanical & 0.016 & 0.093 \\
\hline Excel Expertise & 3.65 & 1.50 \\
\hline Knowledge & 3.76 & 1.71 \\
\hline Difficulty & 3.50 & 1.76 \\
\hline Effort & 5.80 & 1.26 \\
\hline Experience & 1.54 & 0.81 \\
\hline Error Aware & 0.46 & 0.48 \\
\hline Male & 0.52 & 0.49 \\
\hline \multicolumn{2}{|l}{} \\
\hline
\end{tabular}

Overall, respondents thought that their Excel Expertise was slightly below average, average being the midpoint on the seven-point scale ranging from Novice to Expert. Similarly, respondents rated their domain knowledge for this task as slightly below average. And the task's difficulty was also rated below average indicating that the majority of respondents did not find the task overly difficult. The Experience mean of 1.54 shown in Table 1 indicates that the majority of respondents indicated that they had accumulated between 50 and 100 hours of experience with Excel.

Of all respondents, $46 \%$ had experienced the Error Awareness approach. Obviously the remaining 54\% had been trained using the Structured Design approach. Males comprised 52\% of the respondents.

Table 2 contains the results of regression analysis of spreadsheet error rates using the respondent's self-reported Excel Expertise, Knowledge, Difficulty, Effort, and Experience as explanatory variables. Additional explanatory variables were Error Aware, an indicator of having experienced the Error Awareness approach, and respondent's gender. The results reported are the standardized "Beta" coefficients so that differences in explanatory variable scale do not interfere with assessment of the explanatory variable's influence. 
Table 2. Standardized "Beta" Coefficients

\begin{tabular}{|l|c|c|c|c|}
\hline \multirow{2}{*}{$\begin{array}{l}\text { Explanatory } \\
\text { Variables }\end{array}$} & \multicolumn{4}{|c|}{ Error Rates } \\
\cline { 2 - 5 } & All & Omit & Logic & Mechanical \\
\hline Excel Expertise & -0.040 & -0.029 & -0.025 & -0.063 \\
\hline Knowledge & $-0.428^{*}$ & $-0.425^{*}$ & $-0.318+$ & 0.220 \\
\hline Difficulty & 0.076 & 0.009 & 0.106 & 0.190 \\
\hline Effort & 0.118 & 0.025 & $0.333^{* *}$ & $0.236^{*}$ \\
\hline Experience & 0.015 & 0.046 & -0.016 & -0.130 \\
\hline Error Aware & $-0.295^{* *}$ & $-0.266^{* *}$ & $-0.231^{*}$ & 0.016 \\
\hline Male & 0.131 & 0.128 & 0.089 & -0.114 \\
\hline
\end{tabular}

+ Significant at $10 \%$ level, 2-tailed test

$*$ Significant at $5 \%$ level, 2-tailed test

** Significant at $1 \%$ level, 2 -tailed test

Looking at All errors, the results suggest two significant influences, domain knowledge and error awareness. Knowledge of the subject domain being modeled in a spreadsheet results in fewer overall errors. But importantly, having been sensitized to spreadsheet errors also significantly reduces the number of errors.

Since the majority of all errors were Omit errors, it is not surprising that the results are almost identical to those obtained for All errors; that domain knowledge and error awareness significantly reduce error rates. For Logic errors, the situation is slightly different. Domain knowledge and error awareness continue to reduce error rates but strangely, respondents who reported higher effort levels also appear to have made more logic errors. Mechanical errors seem to be more random as the only systematic influence appears to be higher reported Effort levels.

In terms of the hypotheses, these results support both $\mathbf{H}_{\mathbf{1}}$ and $\mathbf{H}_{\mathbf{3}}$ : error awareness and domain knowledge reduce error rates. There is no empirical support for any of the other hypotheses. Rather, there is the somewhat perverse suggestion that increased effort increases Logic and Mechanical errors. After the fact, we may speculate that those respondents who reported greater effort may have been those who had trouble with the task. Those having trouble with the task may well be individuals whose true knowledge of the domain is less than their self-reported knowledge. Similarly, these respondents could have over reported their Excel expertise or underreported their level of difficulty with the task. At this point, we can only speculate about the underlying cause of the unfortunate relationship between effort and Logic and Mechanical errors.

\section{CONCLUSION}

Spreadsheets are a common business tool, frequently used for modeling and decision support. Over the years, numerous studies have consistently found that the error rates in such spreadsheets are alarmingly high. Efforts to control these errors have focused on structural design aspects of the spreadsheets being created (e.g., company standards) and encouraging users to do "code reviews" - to actually look at the formulas they've entered to see if they are correct. As a potential additional tool for controlling spreadsheet errors, this paper introduced a training technique designed to raise students' awareness of problems associated with spreadsheet errors. 
To determine whether or not students sensitized to the risks and consequences of spreadsheet errors would develop spreadsheets with fewer errors, an experiment was designed and conducted. The results were encouraging as students whose spreadsheet training emphasized awareness of spreadsheet errors, developed spreadsheets with fewer errors than other student spreadsheet developers. Additional empirical results suggest that higher reported levels of domain knowledge also significantly reduced spreadsheet error rates. Our results for purely mechanical errors, suggest that some errors occur without any detectable systematic causes.

While the results for the spreadsheet error awareness approach are encouraging, an open question is whether the effect persists. One possibility for future research would be to re-examine error rates at a later date. Does a spreadsheet developer lose a fear of introducing errors over time? Would constant error awareness messages be effective at maintaining in spreadsheet developers a health fear of introducing errors? These questions must remain for future research.

\section{REFERENCES}

1. Benham, H., M. Delaney, A. Luzi. (1993). Structured Techniques for Successful End User Spreadsheets, Journal of End User Computing, 5(2): 18-25.

2. Janvrin, D., J. Morrison. (2000). Using a Structured Design Approach to Reduce Risks in End User Spreadsheet Development, Information \& Management, 37(1): 1-12.

3. Lawrence, R. J., J. Lee. (2004). Financial Modelling of Project Financing Transactions, Institute of Actuaries of Australia Financial Services Forum. The Institute of Actuaries of Australia, Level 7 Challis House 4 Martin Place, Sidney, NSW Australia.

4. Kruck, S.E., J.J. Maher, R. Barkhi. (2003). Framework for Cognitive Skill Acquisition and Spreadsheet Training, Journal of End-User Computing, 15(1): 20-37

5. Morrison, M, J. Morrison, J. Melrose, E.V. Wilson. 2002. A Visual Code Inspection Approach to Reduce Spreadsheet Linking Errors. Journal of End-User Computing, 14(3) 5163.

6. Panko, R.R. (2005). What We Know About Spreadsheet Errors, http://panko.cba.hawaii.edu/SSR/Mypapers/whatknow.htm.

7. Panko, R.R., R.H. Sprague. (1998). Hitting the wall: errors in developing and code inspecting a 'simple' spreadsheet model, Decision Support Systems, 22: 337-353.

8. Simkin, M.G. (1987). How to Validate Spreadsheets, Journal of Accountancy, 164: 130-138. 